\title{
X-ray photofragmentation study of gas-phase indole and indole-water cluster
}

\author{
Thomas Kierspel $^{1,2}$, Joss Wiese ${ }^{1}$, Michele Di Fraia ${ }^{3}$, Rebecca Boll ${ }^{1}$, Cédric Bomme ${ }^{1}$, Benjamin Erk ${ }^{1}$, Daniel \\ Rolles $^{1,4}$, Evgeny Savelyev ${ }^{1}$, Jens Viefhaus ${ }^{1}$, Sebastian Trippel ${ }^{1,2}$, and Jochen Küpper ${ }^{1,2,5}$ \\ 1. Deutsches Elektronen-Synchrotron DESY, Notkestrasse 85, 22607 Hamburg, Germany \\ 2. Hamburg Center for Ultrafast Imaging(CUI), Univ. of Hamburg, Luruper Chaussee 149, 22761 Hamburg, Germany \\ 3. Elettra-Sincrotrone Trieste, Basovizza I-34149, Italy \\ 4. J.R. Macdonald Laboratory, Department of Physics, Kansas State University, Manhattan, KS 66506, USA \\ 5. Department of Physics, University of Hamburg, Luruper Chaussee 149, 22761 Hamburg, Germany
}

The properties of atoms and molecules are strongly dependent on their environment and hydrogen bonds are of universal importance in chemistry and biochemistry. Therefore, it is highly desirable to bridge the gap between single, isolated molecules and molecules in solvation.

Here, we present results on the photofragmentation of indole, the chromophore of the amino acid tryptophan,

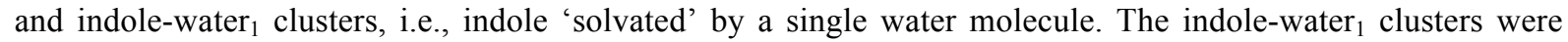
spatially separated from monomeric indole using the electric deflector [1,2]. The photofragmentation was induced by side specific $1 s$ core hole ionization of the indole's nitrogen or carbon atom leading to a relaxation of the highly excited molecules and clusters via an emission of electrons and/or photons and subsequent Coulomb explosion.

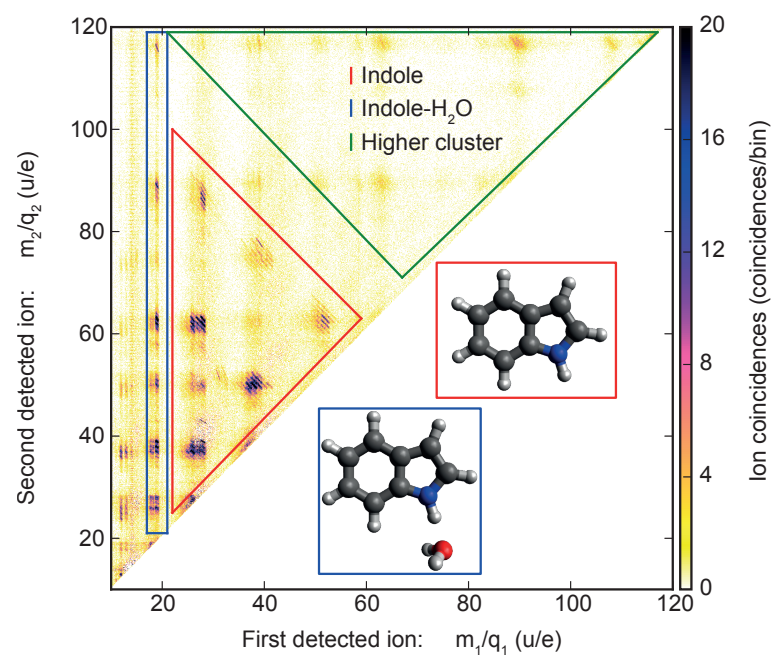

Fig. 1 PIPICO spectrum of the first and second detected ion of indole, indole-water and higher clusters. The different areas in the spectrum are assigned to different species present in the molecular beam.

Photo and Auger electrons as well as ionic fragments were recorded in coincidence with a double-sided velocity map imaging (VMI) spectrometer. The photofragmentation of indole and indole-water was compared by means of (photoelectron-) photoion-photoion coincidence (PIPICO, Fig. 1) maps, i.e., only channels where at least two ionic fragments could be detected have been compared. Charge, proton or hydrogen transfer to the hydrogen bonded water molecule, as well as different Coulomb explosion channels, due to further emission of electrons on the indole-side of the cluster, were observed. The results will be discussed based on the fragmentation channels of the different species, photoelectron and ion VMI images for different ionic fragments, and the 3D reconstruction of the molecule's orientation.

Additionally, we present first results the photophysics following core hole ionization from the oxygen $1 s$ of the hydrogen bonded water. Moreover, we discuss prospects for future time-resolved experiments utilizing UV pump-XFEL probe schemes to unravel the energy flow in UV-excited tryptophan and corresponding peptides.

The indole-water experiment was carried out in a collaboration with DESY and Kansas State University. The experiment was conducted at the Variable Polarization XUV Beamline P04 of PETRA III at DESY.

\section{References}

[1] S. Trippel, Y-P. Chang, S. Stern, T. Mullins, L. Holmegaard, J. Küpper, "Spatial separation of state- and size-selected neutral clusters", Phys. Rev. A. 86, 033202 (2012)

[2] Y-P. Chang, D. Horke, S. Trippel, J. Küpper, "Spatially-controlled complex molecules and their applications", Int. Rev. Phys. Chem. 34(4), 557-590(2015) 